\title{
How to resolve a dilemma about desflurane?
}

Fis or many years Dr. Arnold Patel had used desflurane, a halogenated gas anesthetic, for his cardiac research using dogs. Compared to similar anesthetic gases, desflurane had certain desirable characteristics of importance to Patel's research, such as a higher vapor pressure, better stability against chemical degradation, more rapid uptake and wash out, and negligible metabolism ${ }^{1}$. Patel knew that most university animal facilities used isoflurane for gaseous anesthesia, so when he took a position at Great Eastern University, he brought with him an entire setup for the use of desflurane. The attending veterinarian at Great Eastern had been told that Patel was coming but he did not know that Patel planned on using desflurane. Therefore, when Patel's IACUC protocol application reached him for prereview, he was understandably alarmed because he and his technicians had no clinical experience with desflurane.

At a meeting between Patel, the veterinarian, and the IACUC chairman, Patel apologized for causing a problem but said that before he accepted his new position he had told his new department chairman that he would be using desflurane, and the chairman assured him that would not be a problem. The veterinarian was sympathetic about the lack of communication with the vivarium, but he requested that Patel use isoflurane, because the surgical suite was set up for isoflurane use and the veterinary team was trained on the use of isoflurane. Patel replied that was not possible because all his data and previous publications used only desflurane due to its attributes for his type of research. He suggested that the veterinarian contact the veterinarian at his previous institution to learn what, if anything, Great Eastern should do to accommodate the use of desflurane instead of isoflurane.

The IACUC chairman sided with the veterinarian and said that he believed the committee would not approve the protocol application if Patel did not switch to isoflurane, because it would be considered a matter of safety for the animals. However, as a compromise, he said that Patel should consider purchasing a few additional animals for the veterinary team to train themselves on the use of desflurane.
Patel responded that if that was what the IACUC wanted, it could do that, but the purchase and maintenance of the animals should not be his expense. "Remember," he said, "Great Eastern recruited me. I didn't recruit Great Eastern."

"That may be true," responded the chairman, "but in the past the university has repeatedly said that any needed pilot study or unique training is the financial responsibility of the researcher. In any case, if you want to get your protocol through the IACUC without a substantial delay, you're just going to have to use isoflurane."

How would you approach the problem facing Patel and the IACUC?

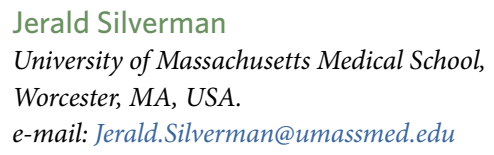

\section{No way to build a relationship...unless animosity is your goal}

\section{T} his scenario from Great Eastern University demonstrates the importance of being agile and innovative when faced with new challenges. A good deal of the Attending Veterinarian's (AV) concerns regarding the use of desflurane could be addressed with a simple phone call to Dr. Patel's previous institution. Depending on where that was located, the $\mathrm{AV}$ at Great Eastern could arrange for Patel's previous veterinarian to visit and train during the first day of surgery. Alternatively, the $\mathrm{AV}$ or vet staff could visit Patel's previous institution. Neither the AWARs nor the PHS policy prevent training in this manner ${ }^{1}$, which would reduce the number of animals needed.

By trying to insist the PI use isoflurane, the AV and IACUC may be missing a great opportunity towards refinement. An article by Lopez et al. that compared different types of inhalation anesthetics suggests that animals sedated with desflurane may have shorter recovery time ${ }^{2}$. Patel has scientific justification for the continued use of desflurane, and, with no indication from the Principle Investigator (PI) that the animals did not fully recover from the desflurane anesthesia, there wasn't a welfare concern with the use of this anesthetic. To mitigate any safety concerns, the BioMedical Safety team should be consulted to ensure desflurane is safe for both people and animals.

If the AV still had concerns about using desflurane, then the purchase of additional animals could be explored. However, the University should provide funds for the training of the veterinary staff. It is the Institute's responsibility to be sure "personnel involved in animal care, treatment, and use are qualified to perform their duties ${ }^{\prime \prime 3,4}$. Specifically, the proper use of anesthetic for any species of animal used by the facility is required by the AWARs.

Due to the lack of communication between the department Chair, IACUC Chair, and AV, Great Eastern University might want to review their processes for on-boarding new PIs. Thankfully, this situation has a few possible solutions to allow the PI's research, as written, to go forward at Great Eastern University.

Rachel Feldman ${ }^{1 凶}$ and Jennifer Shaffer ${ }^{2}$ ${ }^{1}$ Rocky Mountain Laboratories, National Institutes of Health, Hamilton, MT, USA. ${ }^{2}$ Abigail Wexner Research Institute at Nationwide Children's Hospital, Columbus, OH, USA.

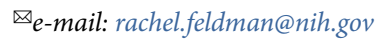

Published online: 25 February 2020 https://doi.org/10.1038/s41684-020-0483-7

References

1. Silverman J., Suckow M., and Murthy S. The IACUC Handbook 3rd Ed. P.486-487 21:5 (CRC Press, Boca Raton, FL 2014).

2. Lopez, L.A., Hofmeister, E.H., Pavez, J.C. \& Brainard, B.M. Am. J. Vet. Res. 70, 1339-1344 (2009).

3. United States Department of Agriculture. Animal Welfare Act and Animal Welfare Regulations 2013.

4. Office of Laboratory Animal Welfare, National Institutes of Health. Public Health Service Policy on the Human Care and Use of Laboratory Animals. (US Department of Health and Human Services, Bethesda, Maryland 2015). 